\title{
Intracranial Germinoma with Leptomeningeal Seeding Along Both Trigeminal Nerves
}

\author{
Jetan Badhiwala, Benedicto Baronia
}

Can J Neurol Sci. 2013; 40: 420-422

Intracranial germinomas (IG) are rare neoplasms. The most frequent primary sites of IG are the pineal and suprasellar regions; however, these tumors commonly disseminate to secondary sites within the central nervous system (CNS) by means of ventricular and subarachnoid cerebrospinal fluid (CSF). The incidence of bilateral Meckel's cave (MC) tumors is exceedingly low. The authors report a case of IG with metastatic involvement of bilateral MC. A review of the literature revealed that this is the first case of an intracranial germinoma - and of any primary CNS tumor - to demonstrate direct leptomeningeal seeding along both trigeminal nerves (CN V).

\section{Case Report}

A 15-year-old boy presented to the emergency department (ED) with a history of facial paresthesias, diplopia, headaches, and emesis. Six weeks prior, he started to develop progressive right-sided facial numbness, with weakness on chewing. Two weeks later, he began to suffer from diplopia; and shortly thereafter, he had onset of intense headaches. He arrived at the ED after a morning episode of vomiting plus headache. At presentation, he reported that his paresthesias had begun to affect the left side of his face. He did not report any hearing loss, bladder or bowel incontinence or retention, or paresthesias or paresis of any of his limbs.

On examination, fundoscopy revealed no papilledema. Diplopia was worse at the lateral extremes of visual gaze. There was decreased sensation to light touch on the right side of the face in the V1 to V3 distribution. The jaw deviated to the right side in the open position, and there was weakness with lateral movement of the jaw. The rest of the neurological exam was normal.

Cranial magnetic resonance imaging (MRI) revealed a nodular mass in the pineal region showing heterogeneous contrast-enhancement (Figure a). There was circumferential thickening of both trigeminal nerves, from the root entry zone (REZ) in the prepontine cistern to Meckel's caves (Figure b). Both CN V exhibited heterogeneous contrast-enhancement. Focal lesions were found in both internal acoustic canals (IAC), though the left-sided lesion was significantly larger in size (Figure c), and on the left aspect of the cervicomedullary junction (Figure d). Spinal MRI revealed several nodular lesions in the lumbar spinal cord and cauda equina (Figure i). A computed tomography (CT) scan of the chest, abdomen, and pelvis was negative for malignancy.

Serum human chorionic gonadotropin (hCG) and alphafetoprotein (AFP) were within normal range: $3 \mathrm{mIU} / \mathrm{mL}$ and 1.7 $\mathrm{ng} / \mathrm{mL}$, respectively. Cerebrospinal fluid hCG and AFP were also within normal range: $2 \mathrm{mIU} / \mathrm{mL}$ and $<0.6 \mathrm{ng} / \mathrm{mL}$, respectively. Cerebrospinal fluid cytology was negative for malignancy/ inflammation.

An open biopsy was performed via partial laminectomy of the L1-L2 vertebrae. We decided to obtain biopsy samples from the spine, rather than the brain, due to the relative ease of access and lower surgical risk. The lesion biopsied was noted to be intradural and extramedullary.

Microscopically, the lesion consisted of sheets of large neoplastic cells with a high nuclear:cytoplasmic ratio and prominent nucleoli. These cells were positive for c-kit (CD117) and placental alkaline phosphatase (PLAP) on immunohistochemistry. Small lymphocytes were interspersed amongst the larger tumor cells. The population of lymphocytes included $\mathrm{CD}^{+} \mathrm{T}$ cells and $\mathrm{CD} 20^{+} \mathrm{B}$ cells. Immunohistochemistry was negative for hCG and AFP. A diagnosis of pure germinoma was made.

The patient was treated with three cycles of carboplatin and etoposide and then 24 Gy of craniospinal irradiation (CSI). Subsequent MRI revealed significant radiological regression of all previously-noted lesions, including the $\mathrm{CN} \mathrm{V}$ involvement (Figure e-h,j). This responsiveness to radiotherapy was consistent with a diagnosis of germinoma.

\section{Discussion}

Intracranial germinomas are malignant tumors, with CSF dissemination occurring in a significant proportion of cases. Metastatic seeding of the leptomeninges encasing the spinal cord is very well-recognized in these neoplasms, and gives rise to classic spinal cord ("drop") metastases.

Our patient had multiple lesions in the lumbar spinal cord and cauda equina. Intraoperatively, these lesions were found to be intradural and extramedullary. Biopsy of one such lesion at the L1-L2 spinal level provided the histological diagnosis of pure germinoma. Most intriguingly, our patient had symmetrical lesions along bilateral trigeminal nerves, extending from the REZ to Meckel's caves.

From the Michael G. DeGroote School of Medicine (JB), Division of Neurosurgery, Department of Surgery (BB), McMaster University, Hamilton, Ontario, Canada.

Received September 26, 2012. Final Revisions Submitted November 26, 2012 Correspondence to: Jetan Badhiwala, Michael G. DeGroote School of Medicine, Michael G. DeGroote Centre for Learning and Discovery, 3101 McMaster University, 1280 Main Street West, Hamilton, Ontario, L8S 4K1, Canada.

Email: jetan.badhiwala@medportal.ca. 


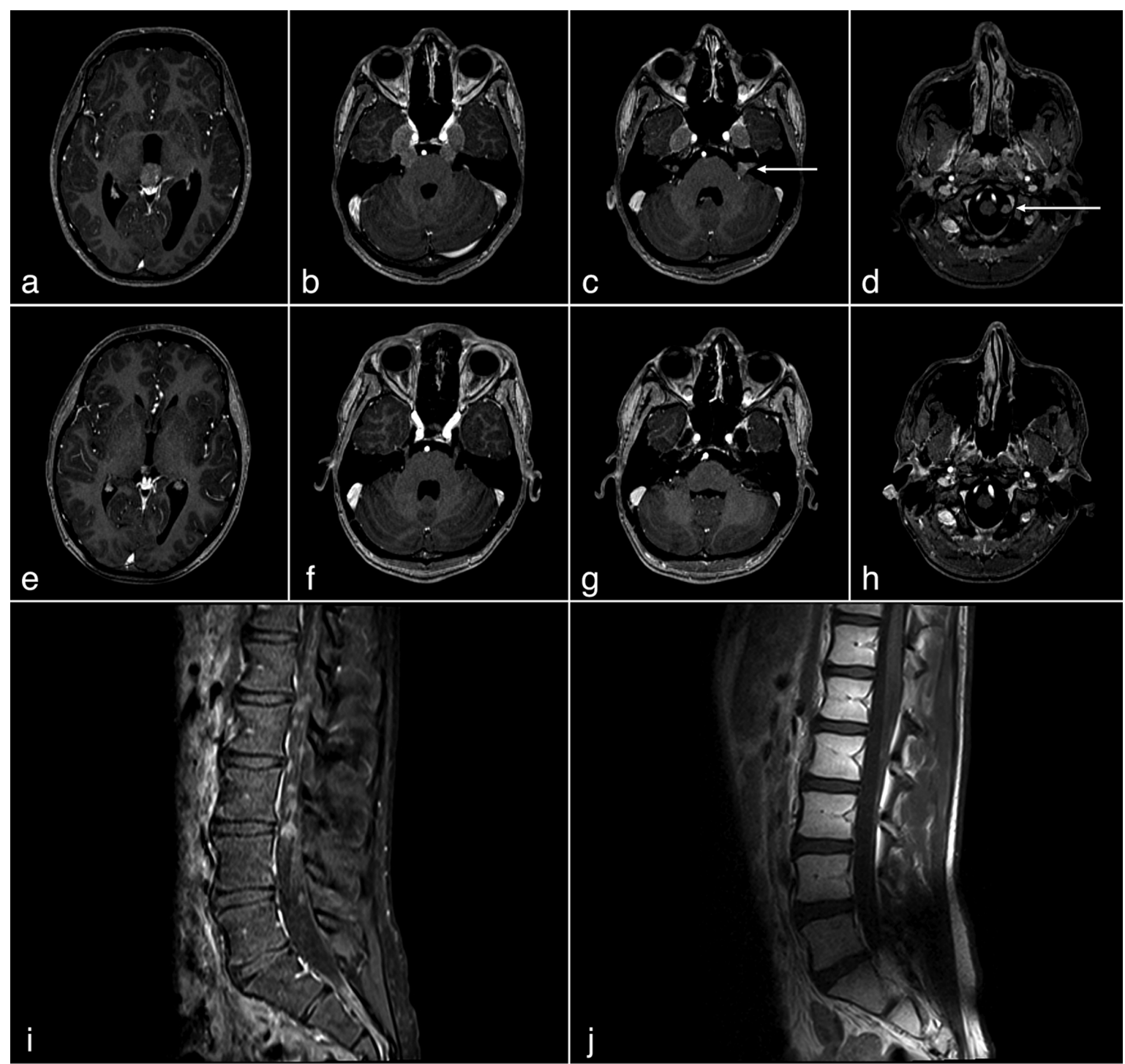

Figure: $(a-h)$ Post-gadolinium $(G d)$ axial head three-dimensional magnetization-prepared rapid acquisition with gradient-echo (3D MPRAGE) MRI. (a-d) A scan at initial presentation revealed several contrast-enhancing lesions: $(a)$ in the pineal region; (b) along both trigeminal nerves, from the REZ to Meckel's caves; (c) in bilateral internal acoustic canals, the left-sided lesion being significantly larger in size (arrow); and $(d)$ at the cervicomedullary junction (arrow). (e-h) Significant radiological regression of each of the above lesions was observed after therapy with 3 cycles of carboplatin and etoposide plus 24 Gy craniospinal irradiation. (i-j) Post-Gd sagittal lumbar spine T1-weighted MRI. (i) A scan at initial presentation revealed several nodular contrast-enhancing lesions in the lumbar spinal cord/cauda equina. (j) A post-therapy scan showed significant radiological regression of disease. Note: the MR image in $(i)$ is with fat suppression, whereas the MR image in ( $j)$ is without fat suppression.

A CT of the chest, abdomen, and pelvis detected no malignancy. The negative CT findings in our patient favored leptomeningeal seeding of intracranial germinoma over hematogenous or perineural spread of a distant cancer as the likely source of the $\mathrm{CN} \mathrm{V}$ lesions. But the possibility that the multiple intracranial tumors seen in our patient arose by independent, multifocal development, rather than leptomeningeal spread of a unifocal tumor, cannot be excluded.

Albeit very rarely, leptomeningeal seeding along the optic nerve (CN II) has been reported in patients with intracranial germinoma ${ }^{1}$. But even then, direct leptomeningeal seeding of an
IG - or of any primary CNS tumor - along the intracranial course of the trigeminal nerve, as found in our patient, has seldom been observed.

Panchawagh et $\mathrm{al}^{2}$ reported leptomeningeal spread of a cerebellar astrocytoma along the right $\mathrm{CN} \mathrm{V}$ root and into Meckel's cave. The patient suffered from headaches, vomiting, vertigo, and ataxia, but unlike in our case, the $\mathrm{CN} \mathrm{V}$ involvement in this patient was clinically occult. In contrast, Maria and colleagues $^{3}$ reported metastatic invasion of the left trigeminal nerve in a case of malignant spinal cord astrocytoma. In this case, the patient was symptomatic for $\mathrm{CN} V$ involvement, with 
left-sided facial numbness; but the primary tumor in the thoracic spinal cord was only found at post-mortem examination. Tubbs and Loukas ${ }^{4}$ described a case of disseminated choroid plexus carcinoma. Metastatic involvement included a lesion extending along the right $\mathrm{CN} \mathrm{V}$ root into $\mathrm{MC}$. The patient suffered rightsided facial numbness and pain. Moreover, Pereira et $\mathrm{al}^{1}$ reported metastatic seeding along bilateral optic nerves in a patient 12 years after treatment of a suprasellar germinoma. There were concurrent lesions along the left trigeminal and facial (CN VII) nerves. While the patient experienced reduced visual acuity in the right eye and left-sided facial paresis, she was asymptomatic for trigeminal nerve pathology. In 2012, Peyre and colleagues ${ }^{5}$ reported a case of multifocal choroid plexus papilloma with a tumor in the fourth ventricle, plus a tumor in the left Meckel's cave, producing left-sided facial hypoesthesia. The authors noted that this multifocal presentation might have been the result of multicentric tumor development or leptomeningeal seeding through subarachnoid spaces.

This is the first report of an intracranial germinoma showing leptomeningeal seeding along both trigeminal nerves, from the root entry zone to Meckel's caves. To our knowledge, it is the first report of bilateral trigeminal nerve seeding in any primary CNS tumor.

\section{Conclusions}

Our patient's earliest and most prominent symptoms were of trigeminal nerve pathology: a six-week history of facial paresthesias with paresis of the muscles of mastication. Headaches and diplopia, both probably attributable to mass effect from the pineal region tumor, only manifested weeks thereafter; and the spinal lesions remained clinically occult. Despite the presence of multiple intracranial tumors and spinal drop metastases, CSF cytology was negative. Moreover, the above symptoms and the Meckel's cave location of tumor may have led to a presumptive diagnosis of trigeminal schwannoma, as reported previously ${ }^{5}$. Hence:

1. The entire neuraxis should be imaged in intracranial germinoma, since even extensive leptomeningeal seeding of the spinal cord may be clinically occult, and further masked by negative CSF cytology.

2. Disseminated intracranial germinoma should be added to the differential diagnosis of bilateral trigeminal schwannoma.

\section{REFERENCES}

1. Pereira LS, Green AJ, Hwang TN, McCulley TJ. Suprasellar germinoma and late perioptic seeding. Eur J Ophthalmol. 2008; 18(1):159-61.

2. Panchawagh J, Muzumdar DP, Goel A. Cerebellar astrocytoma in an adult with extensive leptomeningeal spread. Br J Neurosurg. 2000;14(4):364-6.

3. Maria BL, Cafferty LL, Singer HS, Epstein JI, Carson BS. Diffuse leptomeningeal seeding from a malignant spinal cord astrocytoma in a child with neurofibromatosis. J Neurooncol. 1986;4(2):159-63.

4. Tubbs RS, Loukas M. An unusual finding of the cavernous sinus and Meckel's cave. J Pediatr Neurol. 2007;5(1):53-6.

5. Peyre M, Bah A, Kalamarides M. Multifocal choroid plexus papillomas: case report. Acta Neurochir (Wien). 2012;154(2): 295-9. 